\title{
Correction to: Spinal cord involvement in Kearns-Sayre syndrome: a neuroimaging study
}

\author{
Luca Pasquini ${ }^{1,2}$ - Alessia Guarnera ${ }^{1,2} \cdot$ Maria Camilla Rossi-Espagnet ${ }^{1,2} \cdot$ Antonio Napolitano $^{3}$ - Diego Martinelli ${ }^{4}$. \\ Federica Deodato ${ }^{4} \cdot$ Daria Diodato $^{5} \cdot$ Rosalba Carrozzo $^{5} \cdot$ Carlo Dionisi-Vici $^{4} \cdot$ Daniela Longo $^{1}$
}

Published online: 30 September 2020

(C) Springer-Verlag GmbH Germany, part of Springer Nature 2020

\section{Correction to: Neuroradiology (2020) \\ https://doi.org/10.1007/s00234-020-02501-0}

The original article was published with an incorrect presentation of all author names. The given names and family names were erroneously transposed. All author names are now correctly presented above with given names captured first followed by family name.

Publisher's note Springer Nature remains neutral with regard to jurisdictional claims in published maps and institutional affiliations.

The online version of the original article can be found at https://doi.org/ $10.1007 / \mathrm{s} 00234-020-02501-0$

Luca Pasquini

lucapasquini3@gmail.com

1 Neuroradiology Unit, Imaging Department, Bambino Gesù Children's Hospital, P.zza Sant'Onofrio 4, 00165 Rome, Italy

2 Neuroradiology Unit, NESMOS Department, Sant'Andrea Hospital, La Sapienza University, 00189 Rome, Italy

3 Medical Physics Department, Bambino Gesù Children's Hospital, IRCCS, 00165 Rome, Italy

4 Division of Metabolism, IRCCS Bambino Gesù Children's Hospital, 00165 Rome, Italy

5 Unit of Muscular and Neurodegenerative Disorders, Laboratory of Molecular Medicine, IRCCS Bambino Gesù Children's Hospital, 00146 Rome, Italy 